\title{
An explorative review of youth mental health apps for prevention and promotion
}

\author{
Toni Michel \\ Human Computer Interaction Group \\ TU Wien \\ Vienna, Austria
}

\author{
Petr Slovak \\ Department of Informatics \\ King's College London \\ London, UK
}

\author{
Geraldine Fitzpatrick \\ Human Computer Interaction Group \\ TU Wien \\ Vienna, Austria
}

\begin{abstract}
Youth mental health interventions are increasingly delivered through technology. One factor which may impact engagement and adherence with these apps is how well they fit with young people using them. Yet, how well these technologies fit with requirements and preferences of young people is largely unexplored. By reviewing examples of mental health promotion apps for young people $(n=29)$ we identified a misalignment between young people's media use and what youth mental health promotion apps provide. This presents a space to improve youth mental health technology.
\end{abstract}

\section{CCS CONCEPTS}

\section{- Human-centered computing Interaction}

techniques • Human-centered computing $\sim$ Ubiquitous and mobile devices $•$ Human-centered computing Accessibility technologies

\section{KEYWORDS}

Young people, Mental health promotion, Technology

\section{INTRODUCTION}

Mental health problems during youth (10 to 24 years of age [27]) often persist into adulthood [10], emphasising the importance of early access to mental health promotion and prevention [25]. Promotion improves wellbeing [1], prevention stops or delays the onset of problematic behaviors [1]. Recent evidence suggests that prevention and promotion interventions are the most efficient ways to improve population-level mental health. However, even though young people are the age-group most likely to develop mental health problems, they are also least likely to get help [18].

Permission to make digital or hard copies of part or all of this work for personal or classroom use is granted without fee provided that copies are not made or distributed for profit or commercial advantage and that copies bear this notice and the full citation on the first page. Copyrights for third-party components of this work must be honored. For all other uses, contact the owner/author(s). Pervasive Health, May 2019, Trento, Italy

(๔) 2016 Copyright held by the owner/author(s). 123-4567-24-567/08/06 . . \$15.00 DOI: $10.1145 / 1234$
This gap-between needing and receiving support-stems from two issues: society stigmatizing mental health problems [18] and overwhelmed mental health services wait-listing young people for weeks to months [8]. Stigma impedes young people from accepting they may have problems, and evokes fear of social shaming [18].

Mental health experts advocate for technology to alleviate pressure on services and offer an avenue for support that avoids stigma [16]. Various studies have shown that it is possible to deliver interventions through technology [9] and to prevent harm by doing so [19]. However, recent research points out low uptake, adherence and engagement of young people with mental health technology $[13,8]$. We hypothesize that these issues may be due to neglect of young people's media preferences. Picking up a mental health activity, and sticking with it, is predicated on the activity fitting to the individual [12], and whether youth mental health technology delivers on this fit has not been explored yet.

We investigated our hypothesis by looking into mental health apps. To do so, we collected 29 examples of youth mental health promotion apps and reviewed how they delivered their content. What we found suggests a misalignment between these apps and young people's media preferences. We will now connect our review with a broader research context, explicate in detail what we did and discovered, extrapolate on the implications of our findings, and raise subsequent questions for future research.

\section{RELATED WORK}

Our hypothesis-that there is a mismatch between mental health technology design and young people's expectations-is framed by two major concepts.

1. The role of fit between activity and person

2. Media preferences of young people

Positive Psychology-the discipline strongest focussed on mental health promotion and prevention-increasingly moves from asking "What works?" to "What works for whom?" [21]. This research avenue originally explored questions of fit in relation to activities capitalising on strengths and improving on weaknesses [4]. Lyubomirsky established in 2007 that intrinsic 
motivation increases time and effort invested in mental health activities, which in turn leads to better outcomes [11].

How well an activity fits to a person has been recognized as "critical to the efficacy" of Positive Psychology Interventions [23]. Fit is correlated with higher engagement, adherence and consequently higher wellbeing outcomes [20]. This is consistent with our aforementioned hypothesis that lack of fit may be responsible for low engagement and adherence with youth mental health technology.

As a next step, we turned to data on young people's digital media preferences. Two types were available for this: studies into general media preferences, and attempts to map out attitude and patterns of behavior towards mental health technology in young people.

General youth media preference studies suggest:

- $\quad$ There is a uptake of mobile device use across all age groups from 3 to 15 years old [14].

- Young people prefer highly interactive media, as exemplified by increased video game use [28].

- Young people gravitate towards visually-driven social media platforms (e.g. Instagram) [26].

Youth mental health technology reviews suggest:

- Video conferencing is preferred compared to in-person meetings [2].

- Content should not be static, but dynamic [2].

- Platforms should include social interactions [2].

- Delivery of interventions through games is appreciated but requires game elements to be of high quality [5].

In summary, we found indicators that young people prefer content being delivered through mobile devices, that it is highly interactive and driven by dynamic visual media, and flexible to adjust to their individual needs. We explore how youth mental health apps function, complementing the aforementioned reviews in this space.

\section{METHODS}

We conducted an explorative review of exemplar youth mental health promotion and prevention apps $(n=29)$, looking at how these apps deliver content to young people, and how they can be interacted with.

Mental health apps are complex systems with different aspects reviewers may decide paying attention to. We used Multimodality (MM) as the primary lens; MM means the degree to which modalities-like touch for input, or text for output-are used in conjunction to enrich the interactive depth of a digital system [7]. We had two reasons for this decision: the utilisation of MM for youth mental health systems had not been studied yet, and modalities are the defining characteristics of the youth media preferences indicated in Related work. Therefore, reviewing MM opened up a new avenue of inquiry and a suitable one to investigate our hypothesis.

After collecting the relevant data (see Data collection) we analysed them using the General Inductive Approach. General Inductive Approach "is carried out through multiple readings and interpretations of the raw data" [22], building on the premise that continued engagement with data will eventually allow researchers to recognise patterns and frame assumptions around them. Open and interpretive as it is, General Inductive Approach lends itself well to explorative reviews into new lines of inquiry. There exists precedent for using General Inductive Approach for youth mental health technology reviews [5].

\subsection{Data collection}

We decided on using the same avenues which would be accessible to a young person. First, we queried "mental health" both in Google's Play Store and Apples App Store and noted down all apps that were presented to us. Next, we looked up curated app recommendation lists from prominent youth mental health organisations, like ReachOut[17] and Healthy Young Minds[29] (part of the UK National Health Service). Combined, this got us to 279 entries, including duplicates. Next, we identified the sub-set of those apps that may be considered youth mental health promotion apps. To achieve this, we developed a set of preliminary criteria and rating-system for sorting these apps by who we understood their target audience to be (young person or adults), and what they were aimed at (assessment, prevention, treatment or other). After applying this process, we were left with 29 youth mental health promotion apps.

\subsection{Analyses}

We created rich descriptions of all 29 apps of what they aimed at, how they delivered interventions, and what ways of interaction they afforded. App Store descriptions of the apps, screenshots and user-comments served as a foundation, followed by us installing and manually exploring the apps and taking notes. One researcher then read through these notes looking for how modalities were used to deliver activities, and which activities tended to appear alongside similar modalities, in multiple iterations.

\section{RESULTS}

Overall, the apps we reviewed mostly featured minimalistic functionality: single-tap touch for input, and static images and text for output. There were some examples of more ambitious technology use, like turning the camera into a heartrate sensor, but these were rare.

\subsection{Input}

Single tap touch was the dominant input modality. This held true not only for the basic commands, which is expected, but even for game mechanics (e.g. Happify). Most apps could only be interacted with using single tap touch. Still common, but slightly less so, was the use of written text as input (e.g. 7 Cups of Tea).

Three apps offered taking pictures as input, e.g. Happify featuring a picture-based version of the Three Good Things intervention [30]. Finally, there were two examples of nonminimal input modalities. PAUSE offered a customized version of single tap drag gestures to create an interactive visualization, to calm users down and reduce anxiety. ReachOut Breathe uses the smartphone camera to determine the heart rate of the user 


\subsection{Output}

Written text alongside static images was the prevailing output modality combination and part of all 29 apps. Many apps applied animations to their visualizations, mostly to increase their visual appeal, however in some cases also improving the impact of an intervention. For example, Booster Buddy is using an animated anthropomorphic animal companion, which may increase emotional engagement.

Audio output was part of 11 out of 29 apps (e.g., Headspace). Audio was used both for speech and music, and was mostly present in apps for guided meditation; the only exceptions to this were Recharge, which used audio for an alarm clock function aimed a regulating sleep, and Booster Buddy, which had audio clips playing along the companion's animations.

\subsection{Alignment with youth preferences}

The apps were strongest with respect to including social connectivity. A number of them offered either access to peer communities (e.g. TalkLife) or access to communication with experts (e.g. 7 Cups). A young person looking to engage with peers or experts will likely be able to locate an app allowing it. Communication was predominantly text-based, especially in group-forum types of conversations where postings are visible for everyone. (There may be reasons in terms of moderating content and safeguarding to restrict visual communication in this context.)

The youth mental health promotion apps we reviewed however did largely fail to provide dynamic content; even though young people clearly gravitate towards it, components presented were mostly static. Games are virtually non-existent, despite young people's interest in them.

The apps we reviewed were weakest with respect to customization and choice. Content presentation was mostly predetermined and linear, without choice factoring in. Customization never went beyond minimal cosmetic adjustments (e.g. exchanging the icons of an app). Young people also did not get the chance to impact how they would interact with the apps. Most apps relied primarily on single tap touch and did not offer any alternatives. The same was true for how content was presented. Even though there was some slight variance between apps, as illustrated for example by guidedmeditation apps using voice output, there was never any opportunity given for choice of interaction within an app.

\section{LIMITATIONS}

Current research points to approximately 12,000 existing mental health apps [13]. While it is unclear how many of them would be suitable for young people, it is clear that our review of 29 apps only explored a tiny selection of them. By following app store recommendations and curated lists we focused on apps with a high likelihood of being recommended to help-seeking young. However, it is possible that a review across more youth mental health apps would provide additional insights and expand on our findings, or even contradict us. We hope a broader review will follow this initial exploration.

\section{CONCLUSIONS AND FUTURE WORK}

Our goal was to explore the fit between youth mental health technologies and young people, hypothesizing that many apps do not align with youth media preferences. We have done so by reviewing youth mental health promotion apps, and found data largely consistent with our hypothesis.

By recognizing the importance of fit and designing for it, a range of opportunities open up for improving youth mental health technology. The first opportunity is in improving engagement by exploring increased use of multimodal strategies. Through multimodality, young people could be offered multiple interaction pathways, which in turn would provide them the opportunity to shape the interaction to their individual needs and preferences, thereby potentially increasing engagement. Deciding the most suitable modalities for any given task is something people generally seem to be very proficient at [15].

Additionally, offering different modalities opens new opportunities for research to better understand the relevant mechanism leading to positive outcomes. For example, one of the most potent activities for mental health promotion is the gratitude letter [24], where a person writes a letter to someone detailing all the ways in which the person is grateful for their support. It would also be possible to deliver this activity using video instead of writing a letter by hand. By observing to what degree that shift in modality impacts the effect of the activity, we would learn more about the underlying mechanisms and what part of the activity contributes to its success. Understanding this interplay between modalities and activities may play a significant role in improving the effectiveness of digital mental health interventions in the future.

We propose that mental health promotion apps become more dynamic, with a more sophisticated use of modalities, and also afford young people more choice and customization. This could potentially increase the engagement and adherence of young people with these technologies and contribute to closing the prevention gap, while at the same time providing us with ways to better understand why interventions have the effect they do, which, in turn, will help us in making them better.

\section{ACKNOWLEDGEMENTS}

This research has received funding from the European Union's Horizon 2020 research and innovation programme under Marie Sklodowska-Curie grant agreement No. 722561.

\section{REFERENCES}

1. American Psychological Association. 2014. Guidelines for prevention in psychology. The American Psychologist 69, 3: 285-296. https://doi.org/10.1037/a0034569

2. Katherine M. Boydell, Michael Hodgins, Antonio Pignatiello, John Teshima, Helen Edwards, and David Willis. 2014. Using Technology to Deliver Mental Health Services to Children and Youth: A Scoping Review. fournal of the Canadian Academy of Child and Adolescent Psychiatry 23, 2: 87-99.

3. Helen Christensen, Julia Reynolds, and Kathleen M. Griffiths. 2011. The use of e-health applications for anxiety 
and depression in young people: challenges and solutions. Early Intervention in Psychiatry 5 Suppl 1: 58-62. https://doi.org/10.1111/j.1751-7893.2010.00242.x

4. Lee J. Cronbach and Richard E. Snow. 1977. Aptitudes and instructional methods: A handbook for research on interactions. Irvington, Oxford, England.

5. Theresa Fleming, Mathijs Lucassen, Karolina Stasiak, Matthew Shepherd, and Sally Merry. 2016. The impact and utility of computerised therapy for educationally alienated teenagers: The views of adolescents who participated in an alternative education-based trial: Adolescents views of computerised therapy. Clinical Psychologist 20, 2: 94-102. https://doi.org/10.1111/cp.12052

6. Theresa M. Fleming, Derek de Beurs, Yasser Khazaal, Andrea Gaggioli, Giuseppe Riva, Cristina Botella, Rosa M. Baños, Filippo Aschieri, Lynda M. Bavin, Annet Kleiboer, Sally Merry, Ho Ming Lau, and Heleen Riper. 2016. Maximizing the Impact of e-Therapy and Serious Gaming: Time for a Paradigm Shift. Frontiers in Psychiatry 7. https://doi.org/10.3389/fpsyt.2016.00065

7. Euan Freeman, Graham Wilson, Dong-Bach Vo, Alex Ng, Ioannis Politis, and Stephen Brewster. 2017. The Handbook of Multimodal-Multisensor Interfaces. In Sharon Oviatt, Björn Schuller, Philip R. Cohen, Daniel Sonntag, Gerasimos Potamianos and Antonio Krüger (eds.). Association for Computing Machinery and Morgan \& Claypool, New York, NY, USA, 277-317. https://doi.org/10.1145/3015783.3015792

8. Emily Frith. Access and waiting time in children and young people's mental health services. Retrieved from https://epi.org.uk/wp-content/uploads/2018/01/EPI_Accessand-waiting-times_.pdf

9. Donald M. Hilty, Daphne C. Ferrer, Michelle Burke Parish, Barb Johnston, Edward J. Callahan, and Peter $M$. Yellowlees. 2013. The Effectiveness of Telemental Health: A 2013 Review. Telemedicine and e-Health 19, 6: 444-454. https://doi.org/10.1089/tmj.2013.0075

10. Ronald C. Kessler, Patricia Berglund, Olga Demler, Robert Jin, Kathleen R. Merikangas, and Ellen E. Walters. 2005. Lifetime prevalence and age-of-onset distributions of DSMIV disorders in the National Comorbidity Survey Replication. Archives of General Psychiatry 62, 6: 593-602. https://doi.org/10.1001/archpsyc.62.6.593

11. Sonja Lyubomirsky. 2007. The how of happiness: A scientific approach to getting the life you want. Penguin Press, New York, NY, US.

12. Sonja Lyubomirsky and Kristin Layous. 2013. How Do Simple Positive Activities Increase Well-Being? Current Directions in Psychological Science 22, 1: 57-62. https://doi.org/10.1177/0963721412469809

13. Martha Neary and Stephen Matthew Schueller. 2018. State of the Field of Mental Health Apps. Cognitive and Behavioral Practice 25, 4: 531-537. https://doi.org/10.1016/j.cbpra.2018.01.002

14. Ofcom. Children and Parents: Media Use and Attitudes Report 2017. 306.

15. Sharon Oviatt and Philip R Cohen. 2015. The paradigm shift to multimodality in contemporary computer interfaces.

16. Vikram Patel, Shekhar Saxena, Crick Lund, Graham Thornicroft, Florence Baingana, Paul Bolton, Dan Chisholm, Pamela Y. Collins, Janice L. Cooper, Julian Eaton, Helen Herrman, Mohammad M. Herzallah, Yueqin Huang,
Mark J. D. Jordans, Arthur Kleinman, Maria Elena MedinaMora, Ellen Morgan, Unaiza Niaz, Olayinka Omigbodun, Martin Prince, Atif Rahman, Benedetto Saraceno, Bidyut K. Sarkar, Mary De Silva, Ilina Singh, Dan J. Stein, Charlene Sunkel, and Jürgen Unützer. 2018. The Lancet Commission on global mental health and sustainable development. The Lancet 392, 10157: 1553-1598. https://doi.org/10.1016/S01406736(18)31612-X

17. ReachOut Australia. Tools and Apps. Retrieved January 4, 2018 from https://au.reachout.com/tools-and-apps

18. Debra J. Rickwood, Frank P. Deane, and Coralie J. Wilson. 2007. When and how do young people seek professional help for mental health problems? Medical fournal of Australia 187, 7: S35.

19. Debra Rickwood, Marianne Webb, Vanessa Kennedy, and Nic Telford. 2016. Who Are the Young People Choosing Web-based Mental Health Support? Findings from the Implementation of Australia's National Web-based Youth Mental Health Service. FMIR mental health 3, 3: e40. https://doi.org/10.2196/mental.5988

20. Stephen M. Schueller. 2010. Preferences for positive psychology exercises. The fournal of Positive Psychology 5, 3: 192-203. https://doi.org/10.1080/17439761003790948

21. Stephen M. Schueller. 2014. Person-Activity Fit in Positive Psychological Interventions. In The Wiley Blackwell Handbook of Positive Psychological Interventions. John Wiley \& Sons, Ltd, 385-402. https://doi.org/10.1002/9781118315927.ch22

22. David R. Thomas. 2006. A General Inductive Approach for Analyzing Qualitative Evaluation Data. American fournal of Evaluation 27, 2: 237-246. https://doi.org/10.1177/1098214005283748

23. R. Bruce Thompson, Christine Peura, and William F. Gayton. 2015. Gender differences in the person-activity fit for positive psychology interventions. The fournal of Positive Psychology 10, 2: 179-183. https://doi.org/10.1080/17439760.2014.927908

24. Steven Toepfer and Kathleen Walker. 2009. Letters of Gratitude: Improving Well-Being through Expressive Writing. Journal of Writing Research 1, 3: 181-198. https://doi.org/10.17239/jowr-2009.01.03.1

25. Wahlbeck Kristian. 2015. Public mental health: the time is ripe for translation of evidence into practice. World Psychiatry 14, 1: 36-42. https://doi.org/10.1002/wps.20178

26. 2018. Teens, Social Media \& Technology 2018 Pew Research Center. Retrieved January 13, 2019 from http://www.pewinternet.org/2018/05/31/teens-socialmedia-technology-2018/

27. Adolescence: a period needing special attention recognizing-adolescence. Retrieved January 13, 2019 from http://apps.who.int/adolescent/second-

decade/section2/page1/recognizing-adolescence.html

28. Gaming penetration among children in the U.S. 2015 Statistic. Statista. Retrieved January 13, 2019 from https://www.statista.com/statistics/274600/gamingpenetration-among-children-in-the-us/

29. Healthy Young Minds. Retrieved April 8, 2019 from https://healthyyoungmindspennine.nhs.uk/

30. Three Good Things | Practice / Greater Good in Action Retrieved April 15, 2019 from https://ggia.berkeley.edu/practice/three-good-things 
